Supplement of Hydrol. Earth Syst. Sci., 21, 1251-1262, 2017

http://www.hydrol-earth-syst-sci.net/21/1251/2017/

doi:10.5194/hess-21-1251-2017-supplement

(C) Author(s) 2017. CC Attribution 3.0 License.

(c) (i)
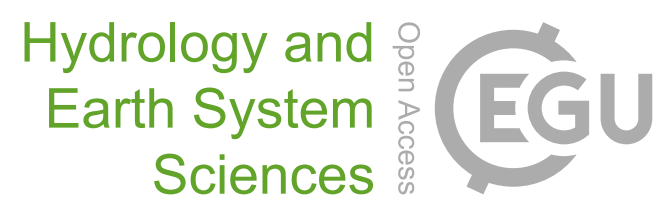

Supplement of

\title{
On the coupled unsaturated-saturated flow process induced by vertical, horizontal, and slant wells in unconfined aquifers
}

Xiuyu Liang et al.

Correspondence to: Xiuyu Liang (xyliang@nju.edu.cn) and Hongbin Zhan (zhan@geos.tamu.edu)

The copyright of individual parts of the supplement might differ from the CC-BY 3.0 licence. 
For the purpose of mathematical convenience, we define the following dimensionless variables,

16

$\mathrm{s}_{\mathrm{D}}=\frac{4 \pi K d}{Q} s, t_{D}=\frac{K}{S_{S} d^{2}} t, x_{D}=\alpha_{x} \frac{x}{d}, y_{D}=\alpha_{y} \frac{y}{d}, z_{D}=\alpha_{z} \frac{z}{d}, x_{0 D}=\alpha_{x} \frac{x_{0}}{d}, y_{0 D}=\alpha_{y} \frac{y_{0}}{d}, z_{0 D}=$

$\alpha_{z} \frac{z_{0}}{d}, \alpha_{x}=\left(K / K_{x}\right)^{1 / 2}, \alpha_{y}=\left(K / K_{y}\right)^{1 / 2}, \alpha_{z}=\left(K / K_{z}\right)^{1 / 2}, K=\left(K_{x} K_{y} K_{z}\right)^{1 / 3}, u_{\mathrm{D}}=$

$18 \quad \frac{4 \pi K d}{Q} u, \kappa_{D}=\frac{d}{\alpha_{z}} \kappa, \beta=\frac{\kappa_{D}}{\sigma}, \sigma=\frac{s_{S} d}{s_{y} \alpha_{z}}, \quad b_{D}=\alpha_{z} \frac{b}{d}$

19 where the subscript $D$ denotes the dimensionless terms. Substituting above dimensionless variables

20 into Eqs. (1)-(4), one obtains the following dimensionless forms of the governing equations for the

21 saturated zone,

22

$$
\frac{\partial^{2} s_{D}}{\partial x_{D}^{2}}+\frac{\partial^{2} s_{D}}{\partial y_{D}^{2}}+\frac{\partial^{2} s_{D}}{\partial z_{D}^{2}}+4 \pi \delta\left(x_{D}-x_{0 D}\right) \delta\left(y_{D}-y_{0 D}\right) \delta\left(z_{D}-z_{0 D}\right)=\frac{\partial s_{D}}{\partial t_{D}}, 0 \leq z_{D}<\alpha_{z},(\mathrm{~S} 2 \mathrm{a})
$$

$$
s_{D}\left(x_{D}, y_{D}, z_{D}, 0\right)=0
$$

$$
\left.\frac{\partial s_{D}}{\partial z_{D}}\left(x_{D}, y_{D}, z_{D}, t_{D}\right)\right|_{z_{D}=0}=0
$$

$$
\lim _{x_{D} \rightarrow \pm \infty} s_{D}\left(x_{D}, y_{D}, z_{D}, t_{D}\right)=\lim _{y_{D} \rightarrow \pm \infty} s_{D}\left(x_{D}, y_{D}, z_{D}, t_{D}\right)=0
$$

26

and for the unsaturated zone,

$$
\frac{\partial^{2} u_{D}}{\partial x_{D}^{2}}+\frac{\partial^{2} u_{D}}{\partial y_{D}^{2}}+\frac{\partial^{2} u_{D}}{\partial z_{D}^{2}}-\kappa_{D} \frac{\partial u_{D}}{\partial z_{D}}=\beta \frac{\partial u_{D}}{\partial t_{D}}, \quad \alpha_{z} \leq z_{D}<\alpha_{z}+b_{D}
$$

and at the interface,

$$
\lim _{x \rightarrow \pm \infty} u_{D}\left(x_{D}, y_{D}, z_{D}, t_{D}\right)=\lim _{y \rightarrow \pm \infty} u_{D}\left(x_{D}, y_{D}, z_{D}, t_{D}\right)=0
$$


and

$$
\begin{gathered}
\frac{\partial^{2} \bar{u}_{D}}{\partial x_{D}^{2}}+\frac{\partial^{2} \bar{u}_{D}}{\partial y_{D}^{2}}+\frac{\partial^{2} \bar{u}_{D}}{\partial z_{D}^{2}}-\kappa_{D} \frac{\partial \bar{u}_{D}}{\partial z_{D}}=\beta p \bar{u}_{D}, \quad \alpha_{z} \leq z_{D}<\alpha_{z}+b_{D}, \\
\left.\frac{\partial \bar{u}_{D}}{\partial z_{D}}\left(x_{D}, y_{D}, z_{D}, p\right)\right|_{z_{D}=\alpha_{z}+b_{D}}=0, \\
\lim _{x_{D} \rightarrow \pm \infty} \bar{u}_{D}\left(x_{D}, y_{D}, z_{D}, p\right)=\lim _{y_{D} \rightarrow \pm \infty} \bar{u}_{D}\left(x_{D}, y_{D}, z_{D}, p\right)=0,
\end{gathered}
$$

43 where $p$ is the Laplace transform parameter and the overbar indicates a variable in the Laplace

44 domain. The Eq. (S5) in a cylindrical coordinate system can be written as following

$$
\bar{s}_{D}-\bar{u}_{D}=0, \quad z_{D}=\alpha_{z}
$$

$$
\frac{\partial \bar{s}_{D}}{\partial z_{D}}-\frac{\partial \bar{u}_{D}}{\partial z_{D}}=0, \quad z_{D}=\alpha_{z}
$$

$$
\frac{1}{r_{D}} \frac{\partial \bar{u}_{D}}{\partial r}+\frac{\partial^{2} \bar{u}_{D}}{\partial r^{2}}+\frac{\partial^{2} \bar{u}_{D}}{\partial z_{D}^{2}}-\kappa_{D} \frac{\partial \bar{u}_{D}}{\partial z_{D}}=\beta p \bar{u}_{D}, \quad \alpha_{z} \leq z_{D}<\alpha_{z}+b_{D}
$$

$$
\frac{\partial \bar{u}_{D}}{\partial z_{D}}\left(r_{D}, z_{D}, p\right)=0, \quad z_{D}=\alpha_{z}+b_{D}
$$

$$
\bar{u}_{D}\left(\infty, z_{D}, p\right)=0 .
$$
boundary condition Eq. (S7c) will be (Rezaei et al., 2016):

$$
\bar{u}_{D}\left(r_{D}, z_{D}, p\right)=\sum_{n=0}^{\infty} \frac{8 \cos \left(\omega_{n} z_{0 D}\right)}{p \Psi\left(\omega_{n}\right)} K_{0}\left(\Omega_{n}\left|r_{D}-r_{0 D}\right|\right) \mathcal{H}_{n}\left(z_{D}, p\right) .
$$

Substituting Eq. (S8) into Eq. (S7) yields:

2

$$
\begin{gathered}
\frac{\partial^{2} \mathcal{H}_{n}}{\partial z_{D}^{2}}-\kappa_{D} \frac{\partial \mathcal{H}_{n}}{\partial z_{D}}-\left(\beta p-\Omega_{n}^{2}\right) \mathcal{H}_{n}=0, \quad \alpha_{z} \leq z_{D}<\alpha_{z}+b_{D} \\
\frac{\partial \mathcal{H}_{n}}{\partial z_{D}}\left(z_{D}, p\right)=0, \quad z_{D}=\alpha_{z}+b_{D}
\end{gathered}
$$




$$
\mathcal{H}_{n}= \begin{cases}e^{M z_{D}}\left[C_{1} e^{N z_{D}}+C_{2} e^{-N z_{D}}\right], & \text { if } \Delta>0, \\ e^{M z_{D}}\left[C_{1} \sin \left(N_{1} z_{D}\right)+C_{2} \cos \left(N_{1} z_{D}\right)\right], & \text { if } \Delta<0, \\ e^{M z_{D}}\left[C_{1} z_{D}+C_{2}\right], & \text { if } \Delta=0,\end{cases}
$$

56 where $M=\kappa_{D} / 2 ; N=\sqrt{\Delta} ; N_{1}=\sqrt{-\Delta} ; \Delta=\kappa_{D}^{2} / 4+\beta p-\Omega_{n}^{2} . C_{1}$ and $C_{2}$ are determined on

57 the basis of Eqs. (S6a) and (S9b), then substituting them into Eq. (S10) yields

$58 \mathcal{H}_{n}= \begin{cases}\cos \left(\omega_{n} \alpha_{z}\right) \frac{(M+N) \exp \left[2 N\left(\alpha_{z}+b_{D}\right)+(M-N) z_{D}\right]-(M-N) \exp \left[(M+N) z_{D}\right]}{(M+N) \exp \left[2 N\left(\alpha_{z}+b_{D}\right)+(M-N) \alpha_{Z}\right]-(M-N) \exp \left[(M+N) \alpha_{z}\right]}, & \text { if } \Delta>0, \\ \cos \left(\omega_{n} \alpha_{z}\right) \exp \left(M z_{D}-M \alpha_{z}\right) \frac{\left[N_{1} \tan \left(N_{1}\left(\alpha_{z}+b_{D}\right)\right)-M\right] \sin \left(N_{1} z_{D}\right)+\left[M \tan \left(N_{1}\left(\alpha_{Z}+b_{D}\right)\right)+N_{1}\right] \cos \left(N_{1} z_{D}\right)}{\left[N_{1} \tan \left(N_{1}\left(\alpha_{Z}+b_{D}\right)\right)-M\right] \sin \left(N_{1} \alpha_{z}\right)+\left[M \tan \left(N_{1}\left(\alpha_{Z}+b_{D}\right)\right)+N_{1}\right] \cos \left(N_{1} \alpha_{z}\right)}, & \text { if } \Delta<0, \\ \cos \left(\omega_{n} \alpha_{z}\right) \exp \left(M z_{D}-M \alpha_{z}\right) \frac{1+M\left(\alpha_{z}+b_{D}\right)-M z_{D}}{1+M\left(\alpha_{z}+b_{D}\right)-M \alpha_{z}}, & \text { if } \Delta=0 .\end{cases}$

\section{S3: Evaluation for eigenvalues $\omega_{n}$}

$$
\begin{cases}-\omega_{n} \tan \left(\omega_{n} \alpha_{z}\right)=\frac{1-\exp \left(-2 N b_{D}\right)}{1 /(\mathrm{M}-\mathrm{N})-\exp \left(-2 N b_{D}\right) /(\mathrm{M}+\mathrm{N})}, & \text { if } \Delta>0 \\ -\omega_{n} \tan \left(\omega_{n} \alpha_{z}\right)=M+\frac{\left[N_{1} \tan \left(N_{1}\left(\alpha_{Z}+b_{D}\right)\right)-M\right] N_{1}-\left[M \tan \left(N_{1}\left(\alpha_{z}+b_{D}\right)\right)+N_{1}\right] N_{1} \tan \left(N_{1} \alpha_{z}\right)}{\left[N_{1} \tan \left(N_{1}\left(\alpha_{z}+b_{D}\right)\right)-M\right] \tan \left(N_{1} \alpha_{z}\right)+\left[M \tan \left(N_{1}\left(\alpha_{Z}+b_{D}\right)\right)+N_{1}\right]}, & \text { if } \Delta<0 \\ \omega_{n}=\left(\kappa_{D}^{2} / 4+(\beta-1) p\right)^{1 / 2} . & \text { if } \Delta=0\end{cases}
$$

64 For $\Delta>0$ the solution domain of $\omega_{n}$ is separated into an infinite series of sub-domain intervals

65 with a period of $\pi$, i.e., $\left(\frac{(2 i-1) \pi}{2 \alpha_{z}}, \frac{(2 i+1) \pi}{2 \alpha_{z}}\right), i=0,1,2, \ldots$. Each solution of $\omega_{n}$ in an individual sub-domain is obtained using the Newton-Raphson method. For $\Delta<0$ the solution domain of $\omega_{n}$ is separated into an infinite series of irregular sub-domains due to the complex formula on the right side of the equation. These irregular sub-domains can be identified by seeking the singular points of function $\mathrm{F}(\omega)=M+\frac{\left[N_{1} \tan \left(N_{1}\left(\alpha_{Z}+b_{D}\right)\right)-M\right] N_{1}-\left[M \tan \left(N_{1}\left(\alpha_{Z}+b_{D}\right)\right)+N_{1}\right] N_{1} \tan \left(N_{1} \alpha_{z}\right)}{\left[N_{1} \tan \left(N_{1}\left(\alpha_{Z}+b_{D}\right)\right)-M\right] \tan \left(N_{1} \alpha_{z}\right)+\left[M \tan \left(N_{1}\left(\alpha_{z}+b_{D}\right)\right)+N_{1}\right]}+\omega \tan \left(\omega \alpha_{z}\right)$, where $\omega$ is a variable 
72 points are separated into an infinite series of irregular sub-domains in which every sub-domain is

73 composed of two adjacent singular points $\left(\omega=\omega_{\mathrm{s}}\right)$ of function $\mathrm{Y}=\mathrm{F}(\omega)$. The $\omega_{n}$ in an

74 individual sub-domain is obtained using the Newton-Raphson method. It is shown in Eqs. (5) and

75 (7) that the saturated zone solution involving the components of unsaturated zone is only

76 represented in the $\omega_{n}$ terms. Changes of the unsaturated zone parameters lead to different $\omega_{n}$

77 values, which affect groundwater flow in the saturated zone.

78

\section{References}

80 Rezaei, A., Zare, M., and Zhan, H. B.: Aquitard Horizontal Dispersion on Reactive Solute

81 Transport in an Aquifer-Aquitard System, Transport Porous Med, 113, 695-716,

$82 \quad$ 10.1007/s11242-016-0719-6, 2016.

83 